\title{
Determinantes del gasto en e-commerce debido a la COVID-19: un análisis empírico para los micronegocios en Monterrey, México ${ }^{1}$
}

\section{Elías Alvarado lagunas*}

\section{RESUMEN}

El objetivo de este artículo es analizar los determinantes que afectan el gasto en que incurren los micronegocios de Monterrey, México, para establecer su modelo de negocio en e-commerce a inicios de la pandemia por la COVID-19. Se emplea una muestra de 661 microempresarios durante el segundo trimestre de 2020. Con la información recabada, se efectúa un modelo Tobit censurado con solución de esquina, el cual permite explicar los factores que influyen en el gasto de una plataforma digital para llevar a cabo el e-commerce de los micronegocios afectados por la pandemia. Los resultados muestran que las características particulares de los propietarios (edad y género) y sus características estructurales (antigüedad, seguridad, clientes y utilidad) impactan significativamente la probabilidad de gastos de adquisición de plataformas digitales, para llevar a cabo el e-commerce de sus productos y servicios.

Palabras clave: e-commerce, micronegocio, COVID-19, modelo Tobit. Clasificación JEL: C01, C13, D13.

Esta investigación ha sido financiada con recursos del Programa de Apoyo a la Investigación Científica y Tecnológica (PAICYT, 2020), como parte del proyecto con folio CSA1311-20. El autor agradece este apoyo, y las valiosas observaciones de los árbitros.

* Profesor-investigador en la Facultad de Contaduría Pública y Administración de la Universidad Autónoma de Nuevo León, México. ORCID: https://orcid.org/0000-0002-2751-7718. Correo electrónico: eliaxalvarado@gmail.com 


\section{ABSTRACT \\ DETERMINANTS OF E-COMMERCE SPENDING DUE TO COVID-19: AN EMPIRICAL ANALYSIS FOR MICRO-BUSINESSES IN MONTERREY, MEXICO}

The objective of this article is to analyze the determinants that affect the spending incurred by the micro-businesses of Monterrey in Mexico, to establish their business model in e-commerce at the beginning of the COVID-19 pandemic. A sample of 661 microentrepreneurs is used during the second quarter of 2020. With the information collected, a censored Tobit model with corner solution is made, which allows to explain the factors that influence the spending of a digital platform to carry out the e-commerce of micro-businesses affected by the pandemic. The results show that the particular characteristics of the owners (age and gender) and their structural characteristics of these businesses (seniority, security, clients and utility) significantly impact the probability of digital platform acquisition expenses, to carry out the $e$-commerce of your products and services.

Keywords: e-commerce, micro business, COVID-19, Tobit model. JEL Classification: C01, C13, D13.

\section{INTRODUCCIÓN}

La pandemia causada por la COVID-19 afecta negativamente a la sociedad y parece que sus efectos se mantendrán, al menos, durante el segundo semestre de 2020. De acuerdo con los datos de la Unesco (Organización de las Naciones Unidas para la Educación, la Ciencia y la Cultura, 2020) 188 países -incluido México- han implementado el cierre de universidades, negocios y empresas no esenciales como respuesta ante la situación generada por la pandemia, lo cual significa que más de $70 \%$ de las personas en el mundo se encuentran en confinamiento.

Al respecto, el Instituto Belisario Domínguez (2020) señaló que el aislamiento y la inmovilización social como medida de disminución y propagación de la COVID-19 conllevan el cierre temporal de empresas no esenciales en todos los niveles (micro, pequeñas, medianas y grandes empresas). En el mismo sentido, Giles (2020) argumentó que este 
evento impredecible trajo consigo la suspensión abrupta de negocios, empresas y sectores económicos, que los obligó a implementar estrategias digitales para seguir ofertando sus productos y servicios bajo las diversas modalidades del e-commerce.

La respuesta de la Facultad de Contaduría Pública y Administración (Facpya) y del Centro de Desarrollo Empresarial (Cedeem) de la Universidad Autónoma de Nuevo León (UANL) a la situación ocasionada por la COVID-19 ha sido brindar asesoría y capacitación virtual a distancia a través de webinars y cursos en línea para apoyar a las empresas a adaptarse a las nuevas formas de negocio en el estado de Nuevo León.

Para el caso específico de los microempresarios ${ }^{2}$ de la ciudad de Monterrey, cabe preguntar ¿qué pasa después de recibir asesoría, capacitación e información sobre las alternativas o modelos de negocio?, ¿realmente estos empresarios están dispuestos a gastar (invertir) en una plataforma de e-commerce?, ¿cuáles son los determinantes que afectan el gasto en que incurren los micronegocios para establecer su modelo de negocio en e-commerce en estos tiempos de la COVID-19? A partir de estas interrogantes, la presente investigación ha analizado el efecto de los factores estructurales y socioeconómicos de los micronegocios respecto al gasto de adquirir una plataforma digitaly de aplicar el e-commerce en el contexto de la situación que enfrenta la sociedad. Entre los factores estructurales del micronegocio se encuentran el tamaño (medido por el número de trabajadores), clientes, sistemas de seguridad y antigüedad; y entre los factores socioeconómicos están algunas características del propietario, como su edad, grado de escolaridad, género y utilidades percibidas en el último año.

Para lograr el objetivo de la investigación y responder a las preguntas planteadas, la Facpya, a través del Cedeem, implementó un cuestionario en línea para los microempresarios o personas autónomas que se registraron para tomar el curso en línea o que están inscritas en la incubadora de negocios de la misma facultad, a través del Observatorio

2 Con el término microempresario nos referimos al empresario que toma decisiones, enfrenta riesgos y obtiene beneficios de una actividad de la cual es el principal responsable. El microempresario puede estar constituido como una persona física o moral y tener hasta 10 trabajadores (INEGI, 2014). Se decide tomar en cuenta solo este perfil en el estudio debido a que la mayoría de las microempresas no poseen una plataforma de venta online como herramienta complementaria en el proceso de comercio electrónico (e-commerce). 
de Competitividad y Desarrollo Empresarial (OCDEM). Este cuestionario fue respondido por 661 microempresarios.

Es conveniente mencionar que, en una primera etapa, esta investigación ha sido financiada por el Programa de Apoyo a la Investigación Científica y Tecnológica (PAICYT) de la UANL. El trabajo consiste en un estudio piloto de corte transversal y coyuntural, y consta de dos etapas. Para llevar a cabo la primera etapa se aplicó un modelo Tobit censurado con solución de esquina, que permitió explicar la relación existente entre el gasto en adquisición de una plataforma digital $-\mathrm{y}$ de esa manera aplicar cualquier modalidad del e-commerce- y las características socioeconómicas y estructurales de los micronegocios. La segunda etapa continuará en 2021 y consistirá en la cuantificación de la percepción y satisfacción de aquellos microempresarios que decidieron obtener (gastar) al inicio de la pandemia una plataforma de e-commerce.

Lo anteriormente expuesto configura el objeto de estudio de esta investigación. De esta manera, la estructura que presenta este documento es la siguiente: en la segunda sección se desarrolla un antecedente de la problemática de la COVID-19 y una breve revisión teórica sobre el problema a estudiar; en la tercera sección se describen la metodología y la forma como se obtuvieron los datos; en la cuarta sección se presentan los resultados obtenidos y, por último, se incluyen algunas conclusiones y recomendaciones pertinentes sobre el tema.

\section{MARCO CONTEXTUAL}

La pandemia ocasionada por la COVID-19 ha afectado negativamente a la sociedad y parece que sus efectos se mantendrán, al menos, hasta el año 2022. De acuerdo con el Diario Oficial de la Federación (2020), los 32 estados de México -incluida la ciudad de Monterrey- han implementado el cierre de actividades académicas, económicas, políticas, sociales y culturales como respuesta ante la pandemia, lo cual significa que más de $80 \%$ de las micro, pequeñas y medianas empresas (MIPyMES) fueron afectadas por la contingencia sanitaria.

Al respecto, el Instituto Belisario Domínguez (2020) argumentó que el aislamiento y la inmovilización social como medida de disminución y propagación de la COVID-19 conllevan el cierre temporal de las actividades económicas (no esenciales) en todos los niveles, lo que traerá como consecuencia una disminución económica en las MIPyMES. En 
el mismo sentido, Lechuga y Hernández (2020) señalaron que este evento impredecible trajo consigo la suspensión abrupta de actividades en los negocios, así como la reducción del comercio, interrupciones en las cadenas de suministros, menor productividad e inversión; es decir, una contracción de la actividad económica en todos los niveles.

Por su parte, el Banco de México (2020) implementó algunas medidas para mitigar el impacto económico en las MIPyMES, entre las cuales destacan la disminución de las tasas de interés, la subasta de dólares para brindar liquidez al mercado y el diseño de un plan de apoyo fiscal a través de la Secretaría de Hacienda y Crédito Público (SHCP). No obstante, autores como Hausmann (2020) y Werner (2020) plantean que la situación económica, a raíz de la pandemia, será aún peor para los países en desarrollo en América Latina -incluido México-, ya que no tienen la misma capacidad de endeudamiento que los países desarrollados y son más propensos a sufrir aumentos en la inflación, caída en el precio de las materias primas, reducción del turismo y reducción del comercio e inversión.

Sin duda alguna, a nivel mundial la pandemia ha provocado la contracción tanto de la oferta como de la demanda en todas las ramas y sectores económicos globales. Sin embargo, Hong y Zhu (2006) y Scupola (2009) señalan que han resurgido otras alternativas de consumo y transacciones de ventas a través del comercio electrónico. Los autores argumentan que las empresas están migrando a las plataformas de e-commerce debido a la practicidad y rapidez de la tecnología web, la cual ha permitido que las empresas se posicionen mejor en los mercados, trayendo consigo mayores utilidades, clientes y socios.

Para el caso de México, Ríos (2020) sostiene que el comportamiento de los consumidores a causa del cierre de las tiendas físicas y todas las medidas sanitarias impuestas por el gobierno federal ha presentado cambios en la compra y venta de productos, y de bienes y servicios. Por ende, se ha manifestado un crecimiento acelerado de comercio electrónico en algunas ramas y sectores económicos del país. Ríos proyecta un crecimiento acelerado en la curva de adopción de compras en línea, la cual alcanzaría una tasa de adopción que se esperaba hasta 2022; empero, debido a la emergencia sanitaria, actualmente se está acelerando con este tipo de transacciones en México. 


\subsection{La COVID-19 y el e-commerce}

Existen una diversidad de estudios que tratan de explicar que el estilo de vida de las personas y empresas ha cambiado en todo el mundo debido a la pandemia originada por la COVID-19. En México, por ejemplo, el aumento en el consumo y ventas a través de internet ha impulsado el crecimiento del e-commerce (OCDE, 2019). La Asociación Mexicana de Ventas Online (2020) señala que, respecto a la percepción y el panorama actual del país a raíz de la pandemia, los consumidores y empresas ven de manera "normal" el cambio de paradigma sobre las compras en línea, motivo por el cual esta asociación proyecta un crecimiento acelerado del e-commerce, ya que los consumidores están recurriendo a la compra de productos e insumos en línea de forma cotidiana y habitual..

Por su parte, Solís y Uruchurtu (2020) señalan que debido a las medidas de contingencia sanitaria impuestas por las autoridades gubernamentales, la gran mayoría de las pequeñas y medianas empresas se vieron obligadas a cerrar temporalmente sus negocios sin contar, al menos al principio del cierre, con estrategias que les brindaran nuevas oportunidades o alternativas de ingresos para solventar económicamente su empresa durante el confinamiento. Los autores argumentan que las nuevas tecnologías y el comercio electrónico son una buena herramienta de apoyo para superar la crisis que originó esta pandemia. Desde algo sencillo, como el uso de redes sociales para difundir y vender sus productos, hasta la búsqueda de plataformas web más desarrolladas, estas aplicaciones prácticas facilitan las ventas en línea para sobresalir durante la contingencia.

En este mismo sentido, la Asociación Mexicana de Internet en Comercio Electrónico (2019) destaca que en los últimos meses el uso de medios digitales en México ha incrementado considerablemente. Por ejemplo, en 2019 se alcanzó un valor de 491 mil millones de pesos, que representó un crecimiento de $24 \%$ respecto a 2017 ; destaca que $85 \%$ de las compras se lleva a cabo vía smartphones y que las redes sociales, principalmente Facebook, permanecen como el medio predilecto de compra y venta en línea.

Por su parte, Méndez (2020) considera que los recientes hechos ocasionados por la pandemia originada por el virus SARS-CoV-2 han demostrado que las finanzas más estables para las personas y empresas son las que se acompañan de las nuevas tecnologías. Establece que, en 
la actualidad, la digitalización de la economía de las empresas, más que una realidad, es una necesidad.

En este sentido, el e-commerce ha adquirido mayor cobertura y popularidad no sólo entre las empresas o corporativos grandes e internacionales, sino también en aquellos micro y pequeños negocios; y es que la facilidad con la que se llevan a cabo las transacciones, sin necesidad de salir de casa o del negocio, hace de esta modalidad de compraventa una de las opciones más seguras y efectivas dentro del mundo económico y financiero.

De esta manera, el e-commerce no sólo ha impactado el mundo económico, sino también la realidad social. A continuación, se describen algunos estudios que nos permiten identificar el e-commerce como un modelo de negocio.

\subsection{El e-commerce como herramienta de ventas en los micronegocios}

En la última década, el desarrollo de la tecnología informática ha generado cambios en diferentes aspectos de la vida social y económica. Las comunicaciones, las transacciones comerciales, el entretenimiento y la forma de relacionarse, entre otros aspectos, se han visto modificados por el uso de internet. La aparición del comercio electrónico (o e-commerce), definido como la venta de bienes y servicios a través de la red, mediante pagos electrónicos, como tarjetas de crédito, constituye el aporte del sector empresarial a esta revolución tecnológica (Zuluaga et al., 2020).

En el mismo sentido, Lee (2001) establece que el e-commerce son todas aquellas transacciones comerciales que se producen a través de internet o la web, que implican un intercambio de valor -como el dinero- y se realizan por medio de las fronteras entre organizaciones o individuos a cambio de productos o servicios.

A su vez, Grandon y Pearson (2004) establecieron que el comercio electrónico puede ser entendido como el proceso de compra y venta de productos y servicios a través de la transmisión de datos por internet y la World Wide Web (WWW). Por su parte, Alam et al. (2008) y Ramanathan et al. (2012) concibieron el e-commerce como una innovación disruptiva en la forma de hacer negocios; argumentan que esta modalidad de negocios representa una gran oportunidad para que las MIPyMES generen valor para que sus clientes se internacionalicen 
(Scupola, 2009); aumenten la eficiencia en las operaciones (Boateng et al. 2008; Ghobakhloo et al., 2011), y realicen transacciones comerciales a través de los medios electrónicos sin tener contacto de forma física o directa (González et al., 2020).

Paralelamente, Iddris (2012), Hernández et al. (2018), Mieles et al. (2018) y Vaithianathan (2010) clasificaron el e-commerce en siete categorías de acuerdo con su aplicación y uso:

- $\mathrm{C} 2 \mathrm{C}$ (Customer to Customer): los consumidores realizan transacciones de manera directa con otros consumidores en la web.

- B2C (Business to Customer): los clientes son atraídos por la publicidad electrónica para adquirir productos y servicios. Este intercambio se realiza mediante sistemas de pago seguro, a través de un envío por un canal físico o electrónico.

- B2B (Business to Business): la transacción de mercado depende de las comunicaciones que se realizan de computador a computador, como un modo rápido, económico y fiable para hacer transacciones de negocio.

- C2B (Customer to Business): transacción en línea en la que los consumidores inician la transacción comercial con las empresas o negocios.

- C2G (Customer to Government): interacción de manera remota en la que los consumidores dan retroalimentación al gobierno a través de sitios web.

- B2G (Business to Government): interacción remota caracterizada porque los negocios retroalimentan al gobierno a través de sitios web.

- G2C (Government to Citizen): interacción remota en la que el gobierno ofrece transacciones o servicios locales, estatales o nacionales (como el pago de impuestos).

En este sentido, se pueden mencionar las teorías de adopción del e-commerce para este estudio. En primera instancia, destaca la Teoría de Difusión de Innovaciones (TDI) de Rogers (1983 y 1995), la cual consiste en estudiar la conducta de adopción de e-commerce en las empresas a través de las fuerzas organizacionales, pues se cree que las características de la empresa -como la antigüedad, experiencia, uso de internet, recursos de Tecnología de Información (TI), número de empleados y rentas- pueden tener un efecto significativo en dicha adopción. También resalta el Modelo de Aceptación Tecnológica o Technology Acceptance Model (TAM) propuesto por Davis (1989 y 
1993), quien indicó que las percepciones del empresario impactan en la decisión de establecer, o no, la modalidad de e-commerce, dado que esta teoría depende de las características del uso de innovación (experiencia) del propietario, así como de los costos percibidos, la dificultad y la imagen representada por el uso del e-commerce.

Igualmente, cabe destacar la teoría del modelo de Tecnología-Organización-Entorno o Technology-Organization-Environment (TOE), propuesta por Tornatsky y Fleischer (1990), quienes aseveraron que la adopción de nuevas tecnologías en los negocios depende de algunas características externas, como la estructura de la economía, la demografía, la infraestructura para la distribución física, el desarrollo de la fuerza de trabajo en Tecnologías de la Información y la Comunicación (TIC), la transparencia y la fiabilidad del sistema financiero y legal del país en el que el negocio reside.

Por otra parte, tanto la Teoría de Acción Razonada o Theory of Reasoned Action (TRA), propuesta por Fishbein y Ajzen (1975) y Ajzen y Fishbein (1980), como la Teoría de Conducta Planeada o Theory of Planned Behavior (TPB), propuesta por Ajzen (1991), indican que los únicos determinantes de la intención son la actitud del dueño de la empresa y la norma subjetiva. Además, se le agrega un tercer factor: la intención de incorporar las TIC como visión de largo plazo y evolución de la empresa.

En suma, las cinco teorías sobre la adopción del e-commerce por parte de las empresas plantean que existen diferentes factores, tanto internos (propios de la empresa) como externos (ajenos a la empresa), que pueden influenciar la adopción de la innovación en estas nuevas formas de hacer negocio. Algunos determinantes son la cultura organizacional de la empresa, los recursos tecnológicos con los que cuenta, las actitudes de la alta gerencia y los cambios estructurales del país (tanto políticos como económicos, sociales y culturales).

El principal objetivo del presente trabajo, como se mencionó en los párrafos introductorios, ha sido analizar el efecto de los factores estructurales y socioeconómicos de los micronegocios respecto al gasto de adquirir una plataforma digital y aplicar el e-commerce en tiempos de la COVID-19. De ahí que la discusión sobre las cinco teorías referidas se configure en el punto de partida de este trabajo. Principalmente, se aborda la referencia de la TDI de Rogers (1983 y 1995) como supuesto para que los dueños o responsables de los micronegocios se percaten de 
que en el contexto de la COVID-19 -sumado a la capacitación y el apoyo que reciben por parte de las universidades del estado de Nuevo León-, el e-commerce es un fenómeno que contribuye al desarrollo empresarial de cualquier organización. Como es natural, el uso de la tecnología se ha vuelto trascendental en la vida cotidiana, pues el acceso a internet y las redes sociales han permitido entablar una estrecha relación de compra y venta que depende de las características de la empresa.

En la Tabla 1 se detallan los estudios realizados en algunos países sobre la adopción del e-commerce en las empresas. Es importante señalar que, de los trabajos descritos en el siguiente cuadro, son pocos los que se relacionan con los gastos en e-commerce en el contexto debido a la contingencia sanitaria. De ahí el interés por generar un aporte en estos temas desde un enfoque empírico.

TABLA 1

REVISIÓN DE LA LITERATURA SOBRE LA ADOPCIÓN DEL E-COMMERCE

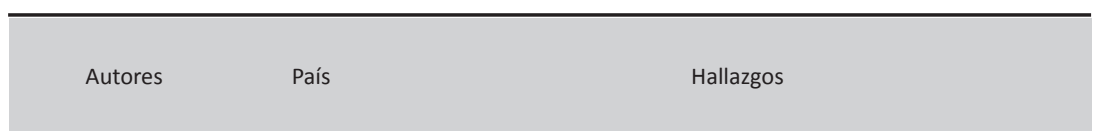

Plantearon un modelo de éxito de e-commerce en el que utilizaron como variable dependiente la satisfacción del cliente en la empresa Molla y Licker (2001) Sudáfrica por el uso de esta modalidad y definieron su relación con la calidad del sistema de e-commerce y la calidad del contenido, uso, confianza y soporte.

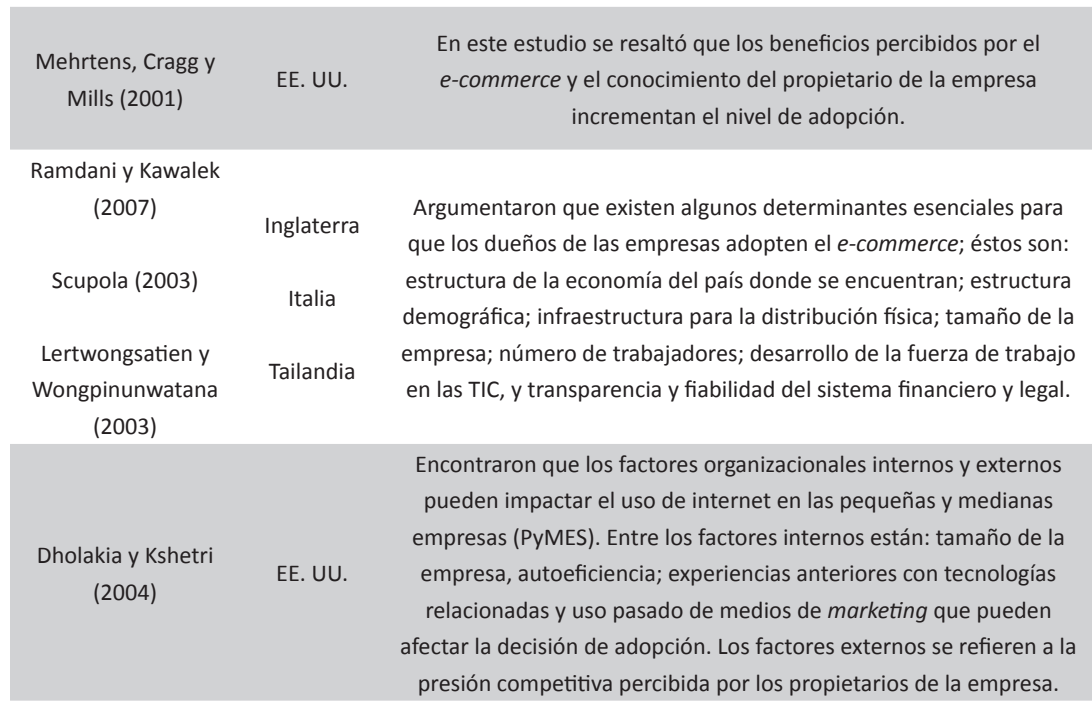


Autores

Utilizaron la TPB para modelar las intenciones, con el fin de adoptar
el e-commerce entre gerentes-propietarios de las PyMES en Chile.
Arandón, Nasco y $\quad$ Chile $\quad \begin{array}{r}\text { Asimismo, encontraron que las dimensiones de norma subjetiva y } \\ \text { actitud predicen positiva y significativamente la intención de adoptar }\end{array}$
las modalidades de comercio electrónico.

Usaron la teoría TOE y concluyeron que es esencial monitorear los factores organizacionales, ambientales y tecnológicos durante Rodríguez y Meseguer (2010) España el proceso de adopción. Incluso, resaltaron la importancia de las condiciones favorables y el oportunismo tecnológico para la adopción del e-commerce.

Señaló que los elementos fundamentales que deben componer el
comercio electrónico son la confianza en los actos de comercio y los
(2015) $\quad \begin{gathered}\text { Magos electrónicos de la empresa o negocio. Asimismo, encontró que } \\ \text { las disposiciones jurídicas que regulan el mencionado comercio en el } \\ \text { país no son claras, pero son importantes para generar confianza tanto } \\ \text { en los dueños como en los clientes. }\end{gathered}$

Estos autores indicaron que las lagunas existentes en la legislación de la informática en México pueden ser un factor decisivo para que

Argüelles (2016)

Alvarado y Robles

(2012)

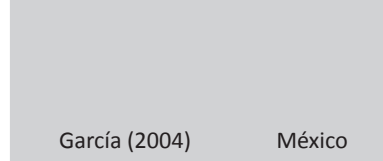

Alvarado y Robles

(2012)

México las empresas no adopten el e-commerce. Algunos motivos para no incentivar su uso pueden ser los siguientes: posibles ciberdelitos; privacidad y protección de los datos personales; seguridad de la información; propiedad intelectual y derechos de autor; nombres de dominios; firmas electrónicas; certificación de documentos; protección del consumidor, etcétera.

Este autor identificó los factores que afectan la implementación del comercio electrónico en México y las soluciones que las empresas han desarrollado. De igual modo, concluyó que, a pesar de que las condiciones económicas mejorarán, pasarán décadas antes de que las personas y los propietarios cambien su comportamiento para adoptar el comercio electrónico ampliamente. El proceso debe ejecutarse poco a poco, porque aún existe desconfianza por parte de ambos agentes económicos.

Estos autores indicaron que las lagunas existentes en la legislación de la informática en México pueden ser un factor decisivo para que las empresas no adopten el e-commerce. Algunos motivos para no incentivar su uso pueden ser los siguientes: posibles ciberdelitos; privacidad y protección de los datos personales; seguridad de la información; propiedad intelectual y derechos de autor; nombres de dominios; firmas electrónicas; certificación de documentos; protección del consumidor, etcétera.

Como consecuencia de la contingencia sanitaria, se acelerará el e-commerce. El autor encuentra que $91 \%$ de las personas que hacen una compra electrónica, realizan una segunda compra en menos de tres meses, tendencia que se mantendrá aún después del levantamiento del confinamiento; este comportamiento se da porque una vez adoptado el hábito de consumo en línea, se convierte en algo recurrente. 
Todas las investigaciones revisadas en este apartado, independientemente de los países, dimensiones o variables analizadas, coinciden principalmente en que el e-commerce ha dado lugar a un cambio paradigmático en el acceso y disposición de la información. De esta manera, los actores (ofertantes y demandantes) que hacen posible este proceso sufren una transformación de actores pasivos a actores activos, y viceversa. En esta revisión de literatura no se encuentra evidencia de trabajos sobre los determinantes que influyen en el gasto de adquisición de una plataforma de e-commerce debido a la COVID-19. Por esta razón, el presente estudio pretende cubrir el vacío de este tipo de análisis en esta temática.

\section{Metodología y dATOS}

\subsection{Obtención de los datos}

Los datos utilizados son de corte transversal y fueron tomados de la Encuesta del COVID-19 y sus efectos en la sociedad, realizada por el Cedeem de la UANL. La encuesta se aplicó en línea durante el periodo marzo y junio de 2020 a través de la plataforma del OCDEM. Se contó con la participación de 661 microempresarios o personas autónomas que se registraron para tomar algún curso en línea o webinars, o que estaban inscritas en la incubadora de negocios de la Facultad de Contaduría Pública y Administración. Del total de la muestra recabada se decidió analizar sólo a los microempresarios de la ciudad de Monterrey, debido a que fue donde se contó con un mayor número de participantes. Aun así, se encontró que algunos microempresarios encuestados no gastaron en la aplicación o adquisición de una plataforma de e-commerce en el periodo de estudio, en cuyo caso se les asignó un gasto nulo; por esta razón, se aplicó un modelo Tobit, que se expondrá con más detalle en la sección "Especificación del modelo empírico".

Cabe aclarar que la encuesta está dividida en cuatro secciones con las cuales se profundizó en algunos temas concretos, como características de los microempresarios; situación económica; gastos en prevención y percepción acerca de los efectos de la pandemia en la sociedad. La primera sección contiene información sobre las características sociodemográficas de los dueños de los micronegocios, número de trabajadores y aspectos de la actividad económica a la que están vinculados. En la segunda parte se habla sobre los ingresos y gastos por su actividad 
económica; en la tercera se presenta la información de los gastos relacionados específicamente por la pandemia; y en la cuarta sección se recaba la información pertinente sobre la percepción de los efectos de la contingencia sanitaria en la sociedad.

\subsection{Descripción de las variables}

Para alcanzar el objetivo propuesto en esta investigación e identificar los determinantes que afectan el gasto de los microempresarios para adquirir una plataforma de e-commerce, se analizaron algunas características estructurales y socioeconómicas de los micronegocios, tal como se observa en la Tabla 2.

TABLA 2

DESCRIPCIÓN DE VARIABLES

\begin{tabular}{|c|c|}
\hline Variables & Descripción \\
\hline Gasto & $\begin{array}{l}\text { Gasto que el microempresario realizó para la adquisición de la } \\
\text { plataforma de e-commerce en pesos mexicanos. }\end{array}$ \\
\hline Edad & Edad del dueño o responsable del micronegocio. \\
\hline Género & Variable dummy: 1 = Hombre, 0 = no. \\
\hline Escolaridad & Nivel de formación académica en años. \\
\hline Antigüedad & Número de años que lleva activo el micronegocio. \\
\hline Empleados & Número de trabajadores en el micronegocio. \\
\hline Clientes & Promedio mensual de clientes que frecuentan el micronegocio. \\
\hline Seguridad & $\begin{array}{c}\text { Variable dummy: } 1 \text { = si el micronegocio cuenta con cámaras y alarmas } \\
\text { de seguridad, } 0=\text { no }\end{array}$ \\
\hline Utilidad & Rendimiento promedio mensual del micronegocio en el último año. \\
\hline
\end{tabular}

Fuente: elaboración propia.

El análisis descriptivo de las variables mencionadas se muestra en la Tabla 3, donde se aprecia que 36.9\% de los microempresarios argumentó gastar o invertir en plataformas digitales para llevar a cabo las ventas de sus productos y servicios en la modalidad de e-commerce. Como resultado, el monto promedio fue de 5,193 pesos, específicamente este gasto se dio en aquellos micronegocios que tienen más de dos años en operación (2.13). Asimismo, se aprecia que la edad promedio de los microempresarios participantes en este estudio fue de 44.9 años.

Respecto a la muestra recabada, los microempresarios del sexo masculino fueron quienes más accedieron a contestar la encuesta (64 $\%$ ); la mayoría señaló que tiene a su cargo, en promedio, a dos personas 
que trabajan en el micronegocio (1.58). Es importante señalar que el grado promedio de escolaridad alcanzado en la muestra fue de 11.6 años, lo que equivale a tener una carrera técnica o estudios de educación media superior.

TABLA 3

ESTADÍSTICOS DESCRIPTIVOS DE LAS VARIABLES

\begin{tabular}{ccccc}
\hline Variables & Media & Desviación & Mínimo & Máximo \\
\hline Gasto & 5193.07 & 7405.29 & 0 & 25326.0 \\
\hline Edad & 44.93 & 8.02 & 30 & 60 \\
\hline Género & 0.64 & 0.48 & 0 & 1 \\
\hline Escolaridad & 11.62 & 2.79 & 6 & 16 \\
\hline Antigüedad & 2.13 & 1.11 & 1 & 8 \\
\hline Empleados & 1.58 & 1.47 & 0 & 112 \\
\hline Clientes & 63.76 & 26.27 & 18 & 1 \\
\hline Seguridad & 0.81 & 0.39 & 0 & 48000 \\
\hline Utilidad & 11733.69 & 6295.58 & 1750 & 8 \\
\hline
\end{tabular}

Fuente: elaboración propia.

Una característica intrínseca de los datos de gastos de inversión de este tipo es que todas las observaciones sólo deben tener valores mayores que 0 . Por esta razón, en muchos casos se presenta una asimetría positiva, debido a que se encuentra una proporción importante de los datos en 0 . Sin embargo, en este estudio, 417 de los 661 micronegocios, equivalentes a $63.1 \%$ de las observaciones, no realizaron ningún tipo de gasto en e-commerce pese a que recibieron asesorías y cursos de capacitación sobre la importancia de vender y ofrecer sus productos y servicios por medio de plataformas de comercio electrónico. Además, es necesario destacar que existe una cantidad considerable de casos dentro de la muestra que tienen valores de gasto cercanos a 0 , lo que refuerza la asimetría positiva de los datos, tal y como se observa en la Figura 1. 
FIGURA 1

DISTRIBUCIÓN DEL GASTO DE MICROEMPRESARIOS POR E-COMMERCE

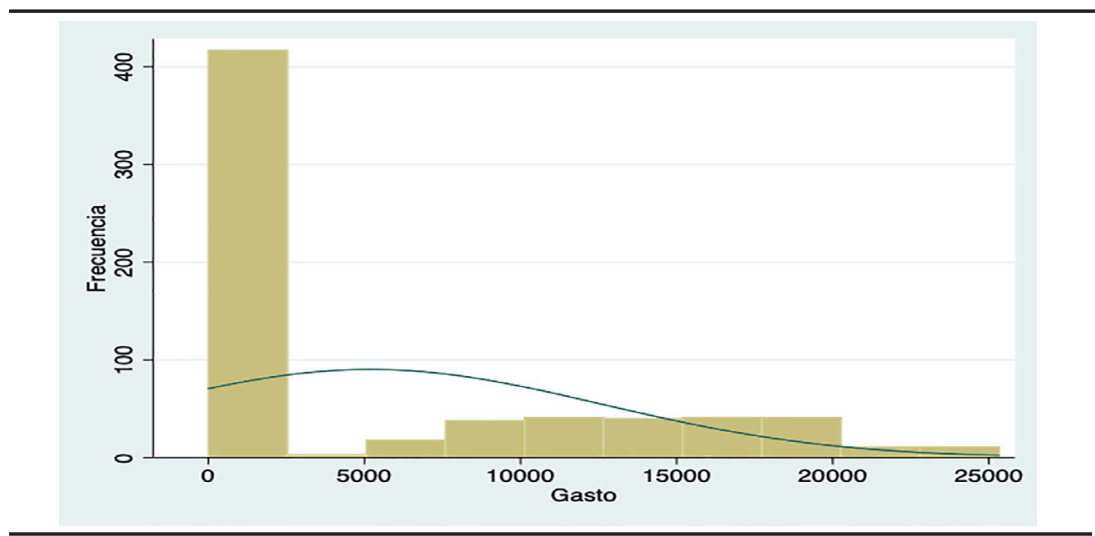

Fuente: elaboración propia.

\subsection{Especificación del modelo empírico}

La estrategia empírica de este estudio se basó en los modelos de solución de esquina y regresiones con censura expuestos por Wooldridge (2002). Este tipo de solución indica que la estructura de los datos -como en este caso- hace que parezca que existe un inferior: $41.4 \%$ de los valores de gasto reportados por los microempresarios se encuentran en 0 . Pero hablar de censura para este análisis no es lo más adecuado, debido a que, en efecto, se aprecia que los valores reportados por los dueños de micronegocios no son un problema de observabilidad, sino de decisión. En esta misma línea, Pérez (2005) llamó a las variables que presentan estas características "Resultados de solución de esquina", pues son valores que escogen las personas y que maximizan su utilidad, por lo que el modelo usado debería llamarse Modelo de solución de esquina y no de censura.

En ese sentido, en este artículo se presenta el caso particular de censura de datos, que surge cuando la variable dependiente vale 0 para una parte de los microempresarios encuestados y, en contraposición, es continua para los valores positivos. Por consiguiente, el modelo presente en este trabajo es de variable limitada con solución de esquina, concretamente un modelo Tobit censurado con solución de esquina.

En datos de este tipo, como lo señaló Wooldridge (2002), no se deben emplear los modelos clásicos - como el de mínimos cuadrados ordinarios (MCO)-, dado que la gran cantidad de observaciones que 
están concentradas en 0 pueden traer sesgo de atenuación en las estimaciones. No obstante, Greene (2002) argumentó que se puede realizar una estimación al restringir los datos únicamente a las personas que realizaron el gasto, para así obtener un resultado aproximado.

Al tomar en cuenta lo anterior, junto con las características de los datos, se pretendió conocer qué tanto afecta la decisión de los microempresarios en el gasto en que incurren en plataformas de e-commerce ante el evento de la pandemia causada por la COVID-19. Para ello, se utiliza un modelo Tobit con censuramiento o Tobit tipo I, que funciona en situaciones en las que el resultado es de selección de esquina como éste. Dicho modelo permite evaluar una única decisión por parte de los microempresarios: el nivel de gasto en que incurrieron debido a la contingencia sanitaria.

Ahora bien, el modelo general en el que existe censura y no una solución de esquina es el siguiente:

$$
Y=X \beta+\varepsilon_{i}, \text { en donde } \varepsilon_{i} \sim \operatorname{Normal}\left(0, \sigma^{2}\right)
$$

El modelo se calcula por Máxima Verosimilitud $(M V)$ e indica qué determinantes influencian el gasto, así como sus valores esperados; por ende, puede considerarse un modelo de variable latente (Pérez y Moral, 2015). Sin embargo, en este caso no aplica, $E\left[Y^{*}\right]=X \beta$, dado que en todo momento se observa el valor gastado en plataformas de e-commerce (Y) y no una construcción de éste. Así pues, es oportuno mencionar lo referido por Wooldridge (2002), que el resultado buscado en los modelos de solución de esquina es $E(Y \mid X, Y>0)$, siempre y cuando sólo se deseen conocer los resultados de los que efectivamente realizaron un gasto mayor que cero; en cambio, al usar $E(Y \mid X, Y)$-este último es el que se analiza en este estudio- se busca conocer el resultado para todas las observaciones disponibles.

Por su parte, Pérez (2011) estableció que en los modelos con censura el $\beta$ brinda la información necesaria en las estimaciones, i.e., $E\left(Y^{*} \mid X\right)=X \beta$. Pero en este estudio se aplicó un modelo de solución de esquina por la ausencia de linealidad en los parámetros, debido a que se pretendió identificar el efecto de los determinantes de los microempresarios sobre el gasto en plataformas de e-commerce como consecuencia de la pandemia. Por lo tanto, la no linealidad de los parámetros y el 
gasto por e-commerce se predice dependiendo de las características de los micronegocios:

$$
E(Y \mid X)=\varnothing\left(\frac{X \beta}{\sigma}\right)[X \beta+\sigma \lambda]
$$

Al calcular las derivadas parciales de la ecuación 2 , se obtienen los valores estimados; de ese modo, el resultado sería:

$$
\frac{\partial E[Y]}{\partial x}=\varnothing\left(\frac{X \beta}{\sigma}\right) \beta
$$

Por otro lado, al sacar el inverso de Mills, representado por $\lambda$, equivaldría a $\frac{\varphi\left(\frac{X \beta}{\beta}\right)}{\sigma\left(\frac{X \beta}{\alpha}\right)}$, en el que $\varphi(\bullet)$ y $\varnothing(\bullet)$ son las funciones de densidad y de densidad acumulada, respectivamente.

Conviene resaltar que, en este estudio, el modelo Tobit tipo I se corrió con la transformación logarítmica del gasto para disminuir la dispersión y evitar problemas de heterocedasticidad. Además, se obtiene una distribución que se asemeja más a una normal, como se muestra en la Figura 2.

FIGURA 2

DISTRIBUCIÓN DE LA FUNCIÓN LOGARÍTMICA DEL GASTO DE LOS MICROEMPRESARIOS

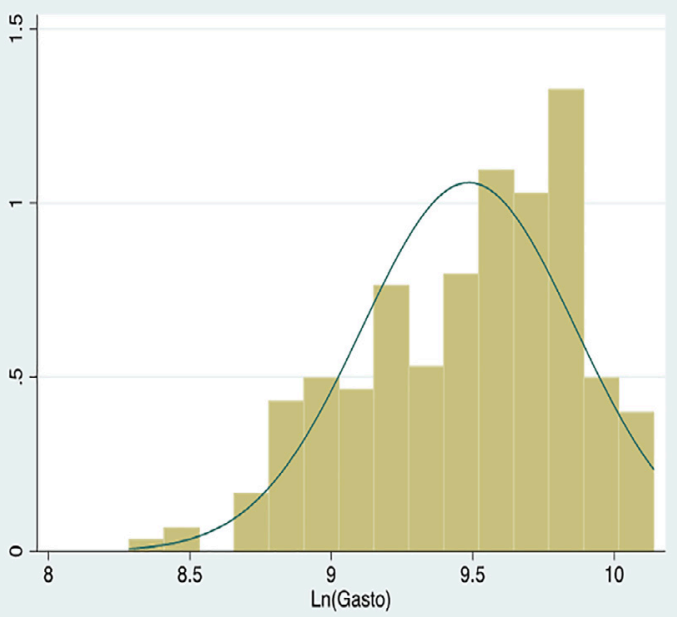

Fuente: elaboración propia. 


\section{RESULTADOS}

En la Tabla 4 se presentan los efectos marginales de los tres modelos Tobit con logaritmo del gasto en e-commerce como variable dependiente, que fueron aplicados en la investigación. ${ }^{3}$ En el primer modelo se incluyen las características estructurales del micronegocio; en el segundo se incorporan las características socioeconómicas del microempresario, y en el tercero se combinan ambas características (estructurales y socioeconómicas) para saber si tienen algún efecto en el gasto de e-commerce.

Después de realizar los modelos, se determinó que las variables socioeconómicas que forman parte de los factores de predisposición -en este caso, el número de empleados en el micronegocio y la edad del dueño- afectan la magnitud del gasto que se realiza para la compra de una plataforma de e-commerce, como se observa en los modelos 1 y 3 . Además, se concluyó que cada año añadido en la edad representa una disminución de $0.02 \%$ en el gasto de una plataforma de e-commerce, mientras que cada empleado adicional en el micronegocio disminuye la probabilidad de gasto en 0.22 por ciento.

Por otra parte, contrario a lo que se esperaba, tener clientes frecuentes en el micronegocio hace que la probabilidad de gastar en una plataforma de e-commerce aumente 0.01 por ciento. Para la interpretación de este resultado se debe tener en cuenta que el hecho de que los clientes sean leales o frecuentes no significa que la atracción de nuevos compradores mejore la probabilidad de gastar en la plataforma digital, dado que su demanda incrementaría. Aunque este resultado es inesperado, puede darse debido a que estos micronegocios tienen una mayor demanda de sus productos y servicios a causa de la contingencia sanitaria.

3 En los tres modelos se aplica el test de razón verosimilitud o likelihood ratio de correcta especificación para conocer cuál de los tres se ajustaba mejor. Asimismo, los modelos se corrieron con una corrección de errores estándar robustos, para tratar de corregir la heteroscedasticidad. 


\section{TABLA 4}

\section{ESTIMACIONES DEL GASTO EN E-COMMERCE DEBIDO A LA COVID-19}

\begin{tabular}{|c|c|c|c|}
\hline Gasto en ecommerce & Modelo 1 & Modelo 2 & Modelo 3 \\
\hline Antigüedad & $\begin{array}{c}0.3381^{* *} \\
(.1371)\end{array}$ & & \\
\hline Empleados & $\begin{array}{c}-0.2234^{*} \\
(.1138)\end{array}$ & & \\
\hline Clientes & $\begin{array}{c}0.0105^{* * *} \\
(.0031)\end{array}$ & & \\
\hline Seguridad & $\begin{array}{l}0.7917^{*} \\
(.4710)\end{array}$ & & \\
\hline Edad & & $\begin{array}{c}-0.0137^{*} \\
(.0071)\end{array}$ & \\
\hline Género & & $\begin{array}{c}-0.4161^{* * *} \\
(.1249)\end{array}$ & \\
\hline Escolaridad & & $\begin{array}{c}-0.00087 \\
(.0241)\end{array}$ & \\
\hline Utilidad & & $\begin{array}{c}0.000025^{* *} \\
(.00001)\end{array}$ & \\
\hline Edad & & & $\begin{array}{c}-0.0195^{*} \\
(.0116)\end{array}$ \\
\hline Género & & & $\begin{array}{c}-0.5722^{* * *} \\
(.2145)\end{array}$ \\
\hline Escolaridad & & & $\begin{array}{l}-0.0044 \\
(.0426)\end{array}$ \\
\hline Antigüedad & & & $\begin{array}{c}0.3115^{* * *} \\
(.1101)\end{array}$ \\
\hline Empleados & & & $\begin{array}{l}0.1508 \\
(.1486)\end{array}$ \\
\hline Clientes & & & $\begin{array}{c}0.0107^{* * *} \\
(.0036)\end{array}$ \\
\hline Seguridad & & & $\begin{array}{c}0.7804^{* *} \\
(.3621)\end{array}$ \\
\hline Utilidad & & & $\begin{array}{c}0.000039 * * \\
(.00002)\end{array}$ \\
\hline Sigma & 4.480 & 4.508 & 4.363 \\
\hline Observaciones & 661 & 661 & 661 \\
\hline $\begin{array}{l}\text { Observaciones } \\
\text { censuradas }\end{array}$ & 244 & 244 & 244 \\
\hline $\begin{array}{c}\text { Observaciones } \\
\text { censuradas-izquierda }\end{array}$ & 417 & 417 & 417 \\
\hline Pseudo-R & 0.01 & 0.01 & 0.03 \\
\hline
\end{tabular}

Los asteriscos $\left({ }^{* *}\right)$ indican significancia estadística de $1 \%,\left({ }^{* *}\right)$ de $5 \%,(*)$ de $10 \%$. El error estándar se encuentra entre paréntesis.

Fuente: elaboración propia. 
En lo que respecta a la antigüedad del micronegocio, se descubrió que tener un año más en el mercado, u operación de compra y venta de productos y servicios, aumenta la probabilidad de gasto en $0.31 \%$, situación que se puede explicar por la mayor estabilidad y lealtad de los clientes de años pasados. Asimismo, otro factor que se esperaba que podía tener algún efecto sobre la cantidad gastada en la plataforma de e-commerce era el grado de escolaridad del dueño del micronegocio; no obstante, resultó no ser estadísticamente significativo. Aunque, para el caso de la variable género, se detalló que tiene un impacto significativo sobre la cantidad gastada en la adquisición de una plataforma de e-commerce; como resultado, los microempresarios de género masculino gastan $0.57 \%$ menos en la adquisición de plataformas de e-commerce respecto a su contraparte femenina.

Igualmente, se observó que los micronegocios que cuentan con seguridad -como cámaras de vigilancia y alarmas- tienden a gastar en la adquisición de una plataforma de e-commerce 0.78 por ciento. De igual manera, cabe resaltar que algunos microempresarios argumentaron tener utilidades antes de la pandemia por la COVID-19 y decidieron realizar el gasto de inversión en una plataforma de e-commerce $(0.000039 \%)$.

\subsection{Contrastes con otros modelos}

Para la correcta especificación del modelo Tobit, propuesta en este estudio, se compararon los resultados del modelo 3 (Tabla 4) con los modelos clásicos en los que insistió Wooldridge (2002) para este tipo de contexto: un MCO y un Probit. Este autor señaló que los resultados de dichos modelos son diferentes, pero mientras no existan grandes cambios entre los resultados ni en la significancia, se puede asumir que el modelo Tobit propuesto no estará mal especificado.

Para el caso del Probit, los estimadores del $\gamma$ deben ser casi idénticos a los del modelo 3 , y se transforman de la siguiente manera: $\gamma=\beta / \sigma$. Por otra parte, en la Tabla 5 se muestran los resultados de los modelos recomendados por Wooldridge (2002), donde se aprecia que dichos modelos comparten significancia y los efectos van en el mismo sentido. Lo anterior quiere decir que las magnitudes de los efectos de cada variable son semejantes entre los modelos, por lo que, a pesar de los indicios de heterocedasticidad que pudiesen existir, el modelo Tobit 
propuesto (modelo 3) está bien especificado y sus estimadores se aproximan a los verdaderos valores.

TABLA 5

COMPARACIÓN DE MCO, PROBIT Y TOBIT

\begin{tabular}{cccc}
\hline \multirow{2}{*}{ Gasto en e-commerce } & MCO & Probit & Tobit \\
& $-0.0186^{*}$ & $-0.0122^{*}$ & $-0.0195^{*}$ \\
Edad & $(.0109)$ & $(.0074)$ & $(.0116)$ \\
& $-0.5569^{* * *}$ & $-.3760^{* * *}$ & $-0.5722^{* * *}$ \\
Género & $(.1568)$ & $(.1058)$ & $(.2145)$ \\
& -0.0034 & -0.0020 & -0.0044 \\
Escolaridad & $(.0267)$ & $(.0184)$ & $(.0426)$ \\
& $0.2478^{* *}$ & $.1886^{* * *}$ & $0.3115^{* * *}$ \\
Antigüedad & $(.1031)$ & $(.0754)$ & $(.1101)$ \\
& 0.1049 & .0891 & 0.1508 \\
Empleados & $(.0860)$ & $(.0621)$ & $(.1486)$ \\
& $0.0094^{* * *}$ & $.0067^{* * *}$ & $0.0107^{* * *}$ \\
Clientes & $(.0028)$ & $(.0020)$ & $(.0036)$ \\
& $0.6428^{* * *}$ & $0.4822^{* * *}$ & $0.7804^{* *}$ \\
Seguridad & $(.1920)$ & $(.1399)$ & $(.3621)$ \\
& $0.000030^{* *}$ & $0.000024^{* *}$ & $0.000039^{* *}$ \\
Utilidad & $(.000012)$ & $(.000009)$ & $(.00002)$ \\
\hline
\end{tabular}

Los asteriscos $\left({ }^{* * *}\right)$ indican significancia estadística de $1 \%,\left({ }^{* *}\right)$ de $5 \%,\left(^{*}\right)$ de $10 \%$. El error estándar se encuentra entre paréntesis.

Fuente: elaboración propia.

Desde el punto de vista académico, el presente trabajo ha pretendido contribuir a los estudios y análisis sobre los efectos e impactos que ha traído consigo la pandemia por la COVID-19 en las empresas mexicanas. La investigación se realiza en la tercera ciudad más importante de México, considerada la médula de la industria mexicana por su gran desarrollo y su diversidad cultural e histórica: Monterrey. Según la investigación, hasta la fecha no se encuentra en la literatura un caso de estudio en el que se utilice un modelo Tobit, con solución de esquina, enfocado en los micronegocios, de ahí el interés de aportar a la investigación de estos temas con esta metodología.

A pesar de que los modelos de elección discreta binaria pueden tener limitaciones en cuanto a representar la variación de preferencia aleatoria -como el caso de las diferencias en las preferencias que no pueden vincularse a las características observadas-, entendemos que conforman un procedimiento válido para representar la variación siste- 
mática de la preferencia; es decir, la variación de preferencia relacionada con las características observadas del decisor. Por tal motivo, reconocemos que la crítica más evidente es que la muestra no es aleatoria, la representatividad no es segura y existen sesgos. Por lo tanto, el trabajo no es comparable y generalizable, de modo que, si se amplía la muestra en otros municipios, la investigación tendría un alcance más representativo.

En este sentido, somos conscientes de las limitaciones y de las oportunidades de mejora; por ejemplo, a nivel conceptual, los resultados han planteado algunas cuestiones que exigen el estudio de otras variables que no han sido analizadas en este trabajo y que podrían mejorar el conocimiento sobre el fenómeno de estudio, como la autoevaluación de capacidades de los microempresarios, las oportunidades económicas, los ahorros, el endeudamiento, así como la realización de un análisis comparativo por género y entre los diferentes municipios del área metropolitana de Monterrey.

\section{CONCLUSIONES}

En esta investigación se demostró que las características particulares de los dueños de los micronegocios (edad y género) y las características estructurales de estos negocios (antigüedad, seguridad, clientes y utilidades) impactan positiva y significativamente la probabilidad de que los microempresarios gasten en la adquisición de plataformas digitales para llevar a cabo el e-commerce de sus productos y servicios. Lo anterior pese a que $60 \%$ de los microempresarios reportó no haber realizado ningún gasto al inicio de la pandemia para la modalidad de e-commerce en sus negocios. Esto es un indicio de que aún existe incertidumbre o desconfianza acerca del comercio electrónico, específicamente en los microempresarios que tienen más de 45 años; sin embargo, se encuentra que los menores de 40 años están dispuestos a realizar ese tipo de gastos, no sólo con el afán de que sus negocios sobrevivan a la pandemia, sino también con el fin de acceder a nuevos mercados y clientes.

Con la aplicación del modelo Tobit censurado con solución de esquina y contrastes con otros modelos fue posible aproximarse a los determinantes actuales del gasto que realizan los microempresarios de la ciudad de Monterrey, México. Y, aunque se encontraron diferencias no despreciables en la magnitud de estos factores que dependen del modelo, se puede concluir que los micronegocios que tienen más tiempo en el mercado (antigüedad) son más estables y generan mayor utilidad; 
por ende, están dispuestos a gastar (invertir) en plataformas digitales para llevar a cabo el desarrollo y la modalidad del e-commerce.

Otro resultado relevante en el estudio fue la relación encontrada entre el gasto realizado por los micronegocios en e-commerce y el gasto en seguridad. De dicha relación se puede inferir que aquellos micronegocios que cuentan con sistemas de seguridad -como cámaras de vigilancia, circuito cerrado y alarmas- fueron los que realizaron un mayor gasto en plataformas de e-commerce para poder mantener y acceder a más clientes.

A partir de los hallazgos de este trabajo es pertinente someter a consideración las siguientes recomendaciones de política pública para fortalecer los micronegocios como fuentes generadoras de empleo e ingreso para la ciudad de Monterrey:

-Las universidades y autoridades municipales, e incluso estatales, encargadas de promover la autosuficiencia económica y el desarrollo empresarial en el estado de Nuevo León, deben trabajar de manera conjunta para llevar a cabo acciones que garanticen el entendimiento y comprensión de las nuevas modalidades de sobrevivencia y negocio en el e-commerce.

- Establecer convenios con la banca, cadenas comerciales e instituciones del gobierno para ofrecer pequeños créditos o financiamientos accesibles, tanto en tasas de intereses como en plazos, con el objetivo de adaptar y obtener plataformas digitales de e-commerce. En este sentido, las universidades podrían ayudar en la capacitación para la autoadministración y la lectura de dicha herramienta digital en los pequeños negocios.

\section{BibliografíA}

Ajzen, Icek (1991), "The theory of planned behavior", Organizational Behavior and Human Decision Processes, 50, Northwestern University, Evanston, Illinois, EE. UU., pp. 179-211.

Ajzen, Icek y Martin Fishbein (1980), Understanding attitudes and predicting social behavior, Englewood Cliffs, NJ, Prentice-Hall.

Alam, Syed Shah, Ali Khatibi, Mohd Ismail Sayyed Ahmad y Hishamuddin Bin Ismail (2008), "Factors affecting e-commerce adoption in the electronic manufacturing companies in Malaysia”, International Journal of Commerce and Management, 17(1/2), Department of Management Studies, Gurunanak Institute of Engineering and Technology, Kalmeshwar Road, Dahegaon, Nagpur, 
Maharashtra, India, pp. 125-139.

Alvarado, Laura Margarita y Celestino Robles (2012), "La percepción acerca de la privacidad y seguridad en el social commerce en México: Un estudio exploratorio", Red Internacional de Investigadores en Competitividad, 6(1), Universidad de Guadalajara, México, pp. 634-646.

Argüelles, María del Consuelo (2016), "Retos de la legislación informática en México", Computación y Sistemas, 20(4), IPN, México, pp. 827-831.

Asociación Mexicana de Internet en Comercio Electrónico (2019), "Comercio electrónico en México". <https:/www.asociaciondeinternet.mx/comercioelectronico-en-mexico>

Asociación Mexicana de Ventas Online (AMVO) (2020), "Estudio de Venta Online, Versión pública". <https:/www.amvo.org.mx/estudios/estudio-sobreventa-online-en-mexico-2020/>

Banco de México (Banxico) (2020), “Anuncio de la Comisión de Cambios. Se incrementa el tamaño del programa de coberturas cambiarias liquidables en moneda nacional que subasta el Banco de México". <https://www.banxico. org.mx/publicaciones-y-prensa/anuncios-de-la-comision-de-cambios/comunicados-comision-cambios.html $>$

Boateng, Richard, Richard Heeks, Alemayehu Molla y Robert Hinson (2008), "Ecommerce and socio-economic development: conceptualizing the link", Internet Research, 18(5), University of London, Inglaterra, pp. 562-594.

Davis, Fred (1989), "Perceived usefulness, perceived ease of use and user acceptance of information technology", MIS Quarterly, 13, Management Information Systems Research Center, University of Minnesota, pp. 319-340.

Davis, Fred (1993), "User acceptance of information technology: Systems characteristics, user perception and behavioral impacts", International Journal of Man-Machine Studies, 38, Academic Press, United Kingdom, pp. 475-487.

De los Ángeles, Alma (2015), “Análisis y perspectivas del comercio electrónico en México", Perfiles de las Ciencias Sociales, 3(5), División Académica de Ciencias Sociales y Humanidades, UJAT, México, pp. 61-87.

Dholakia, Ruby Roy y Nir Kshetri (2004), "Factors impacting the adoption of the Internet among SMEs", Small Business Economics, 23(4), Springer Science, EE. UU., pp. 311-322.

Diario Oficial de la Federación (DOF) (2020), "Informe Pleno del Instituto Federal de Telecomunicaciones". <https://www.dof.gob.mx/nota_detalle.php?codigo $=5590787 \&$ fecha $=31 / 03 / 2020 \&$ print $=$ true $>$

Fishbein, Martin y Icek Ajzen (1975), Belief, attitude, intention, and behavior: an introduction to theory and research, Addison-Wesley, Reading, MA.

García, José Heriberto (2004), “La regulación del comercio: retos ante el cambio tecnológico", Revista del Instituto de Ciencias Jurídicas de Puebla, 12(41), Instituto de Ciencias Jurídicas de Puebla, México, pp. 43-70.

Ghobakhloo, Morteza, Daniel Arias y José Benítez (2011), “Adoption of e-commerce applications in SMEs", Industrial Management and Data Systems, 111(8), Emerald Group Publishing Limited, United Kingdom, pp. 1238- 1269. Giles, César Alejandro (2020), "Recomendaciones para las MIPyME ¿Qué hacer 
para sobrevivir a la pandemia del Covid-19?”, Notas Estratégicas del Senado de la República, 86, Instituto Belisario Rodríguez, México, pp. 1-13.

González, Marta, Alejandro Lorenzo y Naomi Reyes (2020), "El comercio electrónico en España", Tesis de grado, Universidad de La Laguna, La Laguna, España.

Grandón, Elizabeth, Suzanne Nasco y Peter Mykytyn (2011), “Comparing Theories to Explain E-commerce Adoption”, Journal of Business Research, 64(3), Elsevier Inc., EE. UU., pp. 292-298.

Grandon, Elizabeth y Michael Pearson (2004), "Electronic commerce adoption: an empirical study of small and medium US businesses", Information \& Management, 42(1), Elsevier Inc., EE. UU., pp. 197-216.

Greene, William H. (2002), Econometric Analysis, Prentice Hall, New Jersey.

Hausmann, Ricardo (2020), "Flattening the COVID-19 Curve in Developing Countries, Project Syndicate". <https://www.project-syndicate.org/commentary/flattening-covid19-curvein-developing-countries-by-ricardo-hausmann-2020-03> [25 de enero de 2021].

Hong, Weiyin y Kevin Zhu (2006), "Migrating to internet-based e-commerce: Factors affecting e-commerce adoption and migration at the firm level", Information \& Management, 43(2), University Nevada, EE. UU., pp. 204-221.

Hernández, Sandra, Jorge Hernández y Edgar Olguín (2018), "Comercio electrónico como herramienta complementaria en las PYMES en México", Revista Científica Electrónica de Educación y Comunicación en la Sociedad del Conocimiento, 2(18), Universidad de Granada, España, pp. 1-29.

Iddris, Faisal (2012), "Adoption of E-Commerce Solutions in Small and MediumSized Enterprises in Ghana", European Journal of Business and Management, 4(10), Dean of Business School Amman Arab University, Jordan, pp. 48-57.

Instituto Belisario Domínguez (2020), "Implicaciones económicas de la pandemia por COVID-19 y opciones de política”, Notas Estratégicas del Senado de la República, 81, Instituto Belisario Domínguez, México, pp. 1-11.

Instituto Nacional de Estadística, Geografía (INEGI) (2014), "La micro, pequeña y mediana empresa", Biblioteca de la Micro, Pequeña y Mediana Empresa, 7, Nacional Financiera, México, pp. 24-101.

Lechuga, Mayela del Rayo y Juana Hernández (2020), “La primavera del 2020: Las repercusiones del Covid-19 en los patrones de consumo y el comercio electrónico en Durango, México", Revista de Economía del Caribe, 11, Universidad del Norte, Colombia, pp. 1-16.

Lee, Chung Shing (2001), "An analytical framework for evaluating e-commerce business models and strategies", Internet Research, 11(4), Emerald Group Publishing Limited, United Kingdom, pp. 349-359.

Lertwongsatien, Charlesmsak \& Nitaya Wongpinunwatana (2003), "E- commerce Adoption in Thailand: An Empirical Study of Small and Medium Enterprises (SMEs)", Journal of Global Information Technology Management, 6(3), Taylor, Francis Online, EE.UU., pp. 67-83.

Mehrtens, Jenni, Paul Cragg y Annette Mills (2001), “A model of Internet adoption by SMEs”, Information \& Management, 39(3), Elsevier Inc., EE. UU., pp. 
$165,2001$.

Méndez, Claudia (2020), "El comercio más sano en tiempo de pandemia: e-commerce. Panorama Chile-México", Orientando, 11(21), Universidad Veracruzana, México, pp. 1-18.

Mieles, Jennifer Lisbeth, Andrea Daniela Albán, Jaritza Briced Valdospin y Dayana Elizabeth Vera (2018), "E-commerce: un factor fundamental para el desarrollo empresarial en el Ecuador", Revista Cientifica ECOCIENCIA, Universidad Tecnológica ECOTEC de Samborondón, Ecuador, pp. 1-17.

Molla, Alemayehu y Paul Licker (2001), "E-commerce systems success: An attempt to extend and respecify the De- Lone and McLean model of IS success", Journal of Electronic Commerce Research, 2(4), California State University, EE. UU. pp. 131-141.

Organización de las Naciones Unidas para la Educación, la Ciencia y la Cultura (Unesco) (2020), "Impacto de COVID-19 en educación". <https://es.unesco. org/covid19/educationresponse $>$ [25 de enero de 2021].

Organización para la Cooperación y Desarrollo Económico (OCDE) (2019), "Panorama del comercio electrónico. Políticas, tendencias y modelos de negocio". $<$ https://www.oecd.org/sti/Panorama-del-comercio-electro\%CC\%81 nico. pdf $>$

Pérez, César (2005), Métodos estadísticos avanzados con SPSS, Thomson, Madrid. Pérez, César (2011), Econometría Avanzada. Grupo Editorial Garceta, Madrid, España.

Pérez, César e Ignacio Moral (2015), Técnicas de Evaluación de Impacto, Grupo Editorial Garceta, Madrid, España.

Ramanathan, Ramakrishnan, Usha Ramanathan y Hsieh Ling Hsiao (2012), "The impact of e-commerce on Taiwanese SMEs: Marketing and operations effects", International Journal of Production Economics, (140), Elsevier Inc., EE. UU., pp. 934-943.

Ramdani, Boumediene y Peter Kawalek (2007), "SME Adoption of Enterprise Systems in the Northwest of England: An Environmental, Technological and Organizational Perspective", in The International Federation for Information Processing, 235, Organizational Dynamics of Technology-Based Innovation: Diversifying the Research Agenda, Boston, pp. 409-430.

Ríos, Alma de los Ángeles (2020), "Emergencia sanitaria y transacciones electrónicas: Covid-19 caso México", Perfiles de las Ciencias Sociales, 8(15), Universidad Juárez Autónoma de Tabasco, México, pp. 66-82

Rodríguez, Irma y Antoni Meseguer (2010), "Toward a Longitudinal Model of e-Commerce: Environmental, Technological, and Organizational Drivers of B2C Adoption", Information Society, 26(3), Taylor, Francis Online, EE. UU., pp. 209-227.

Rogers, Everett (1983), Diffusion of Innovations, The Free Press, New York, EE. UU., 3rd ed.

Rogers, Everett (1995), Diffusion of Innovations, The Free Press, New York, EE. UU., 4th ed.

Scupola, Ada (2003), "The Adoption of Internet Commerce by SMEs in the South 
of Italy: An Environmental, Technological and Organizational Perspective", Journal of Global Information Technology Management, 6(1), Taylor, Francis Online, EE. UU., pp. 51-71.

Scupola, Ada (2009), "SMEs' e-commerce adoption: perspectives from Denmark and Australia", Journal of Enterprise Information Management, 22(1/2), Taylor, Francis Online, EE. UU., pp. 152-166.

Solís, Anahí y David Uruchurtu (2020), "Las nuevas tecnologías, el comercio electrónico y el diseño como apoyo para superar la crisis económica provocada por Covid-19 en las pequeñas y medianas empresas (PYMES) de Ciudad Juárez, Chihuahua, México", en Jesús Flores, Jacqueline Oyarce y Gloria Rodríguez (coords.), Reflexões sobre internet, tecnologia e comunicação, Universidad Técnica Particular de Loja-Editorial RIA, Ecuador, pp. 250-275.

Tornatsky, Louis y Mitchell, Fleischer (1990), The Processes of Technological Innovation, Lexiton eBooks, Lexiton, MA.

Vaithianathan, Sridhar (2010), "A review of e-commerce literature on Indiaand research agenda for the future", Electron Commer Res, 10, Springer Science, EE. UU., pp. 83-97.

Werner, Alejandro (2020), "COVID-19 Pandemic and Latin America and the Caribbean: Time for Strong Policy Actions”. <https://blogs.imf.org/2020/03/19/ covid-19-pandemic-andlatin-america-and-the-caribbean-time-for-strong-policy-actions/> [24 de enero de 2021].

Wooldridge, Jeffrey (2002), Econometric Analysis of Cross Section and Panel Data, MIT Press, Cambridge.

Zuluaga, Paula, Danny Vargas y Jorge Valencia (2020), "El comercio electrónico B2C y su adopción en el consumidor de la ciudad de Manizales-Colombia”, NOVUM, 1(10), Universidad Nacional de Colombia, Colombia, pp. 10-24. 